\title{
Is It Possible to Prevent Ovarian Hyperstimulation Syndrome by Gonadotropin-Releasing Hormone Agonist Triggering and Modified Luteal Support in Patients With Polycystic Ovarian Morphology?
}

\author{
Ali Sami Gurbuz ${ }^{\mathrm{a}}$, Ruya Deveer ${ }^{\mathrm{b}}$, Mert Kucuk ${ }^{\mathrm{b}}$, Necati Ozcimenª, Dilek Incesu ${ }^{\mathrm{a}}$, Sezen Koseoglu ${ }^{\mathrm{b}, \mathrm{c}}$
}

\begin{abstract}
Background: Gonadotropin-releasing hormone ( $\mathrm{GnRH})$ agonist triggering plus 1,500 IU human chorionic gonadotropin (hCG) supplementation protocol was previously claimed effective in reducing the ovarian hyperstimulation syndrome (OHSS) incidence in high responders.
\end{abstract}

Methods: This retrospective study included women with polycystic ovarian (PCO) morphology who were at high risk of OHSS and were given the GnRH agonist trigger plus hCG luteal support protocol in a single center.

Results: The mean peak estradiol level was 5,336 $\pm 2,341(1,187$ $19,746) \mathrm{pg} / \mathrm{mL}$. The mean number of follicles $>12 \mathrm{~mm}$ on the day of trigger was $22 \pm 7(9-51)$. A total of 88 cycles were undertaken. Sixty-three $(71.5 \%)$ women underwent fresh embryo transfer. Fresh embryo transfer was canceled in $21(23.8 \%)$ and embryo transfer was canceled in four $(4.5 \%)$ women. The overall clinical pregnancy rate was $46.4 \%$ per started cycle. A total of $12(13.6 \%)$ patients developed OHSS. "Freeze-all" policy did not attenuate OHSS in four patients, and three of these patients developed OHSS despite 1,500 IU hCG was not administered.

Conclusion: We conclude that OHSS may still occur with the use of a GnRH agonist trigger combined with low-dose hCG supplementation protocol in women with polycystic ovary syndrome (PCOS) or PCO morphology. Furthermore, we also conclude that "freeze-all" policy also will not completely eliminate OHSS development in high-risk women.

Keywords: OHSS; GnRH agonist; GnRH agonist trigger hCG; "Freeze-all" policy

Manuscript accepted for publication February 23, 2016

aPrivate Novafertil IVF Centre, Konya, Turkey

bDepartment of Obstetrics and Gynecology, School of Medicine, Mugla Sitki Kocman University, Mugla, Turkey

${ }^{\mathrm{c} C o r r e s p o n d i n g ~ A u t h o r: ~ S e z e n ~ K o s e o g l u, ~ D e p a r t m e n t ~ o f ~ O b s t e t r i c s ~ a n d ~ G y-~}$ necology, Mugla Sitki Kocman University Training and Research Hospital, Mugla, Turkey. Email: drsezenkoseoglu@gmail.com

doi: http://dx.doi.org/10.14740/jocmr2500w

\section{Introduction}

Some patients develop excessive response to gonadotropin stimulation during controlled ovarian hyperstimulation $(\mathrm{COH})$ which is the main component of modern in vitro fertilization (IVF) treatment. Certain patient characteristics such as age, body mass index (BMI), ovarian volume, basal follicle stimulating hormone (FSH), inhibin B levels, ovarian blood flow, antral follicle count (AFC), anti-Mullerian hormone (AMH) levels, and polycystic ovary syndrome (PCOS) may help to identify these high-responder patients [1]. Although the definition of high-risk group differs in the literature, patients with PCOS or isolated polycystic ovarian (PCO) morphology characteristics $[2,3]$ are included in the high-risk group. Several approaches have been defined to diminish the risk of an exaggerated response in these high responder patients. Initiating a lower dose of gonadotropin stimulation, withdrawing gonadotropin stimulation for several days ("coasting"), and cryopreservation of all embryos and/or cabergoline treatment were proposed. During antagonist cycles, the use of gonadotropinreleasing hormone $(\mathrm{GnRH})$ agonist to trigger final oocyte maturation is another method recommended to prevent the development of ovarian hyperstimulation syndrome (OHSS) [4-9]. Recently, the use of a GnRH agonist trigger combined with modified luteal support with human chorionic gonadotropin (hCG) in GnRH antagonist cycles has been suggested in highrisk patients with high pregnancy rates $[10,11]$.

At this point, we wanted to present our single center experience using GnRH agonist trigger combined with modified luteal support with 1,500 IU hCG protocol in a group of patients with PCO morphology. There is lack of consensus whether OHSS can be prevented with this protocol [12]. Therefore, the aim of the present study was to investigate whether lower OHSS rates with high clinical pregnancy rates are associated with the use of this protocol in a high-risk group.

\section{Materials and Methods}

This is a retrospective analysis of women who received the GnRH agonist trigger plus 1,500 IU hCG protocol in a GnRH antagonist ovarian stimulation cycle at Private Novafertil IVF Centre, Konya, Turkey between November 2010 and March 
2014. Daily gonadotropin stimulation was started on the second or the third day of either a spontaneous or an induced menstrual cycle; the starting dose was determined (ranging from 75 to $300 \mathrm{IU}$ ) according to age, BMI, follicular phase serum FSH level, AFC and previous history of ovarian response if there had been a treatment. GnRH antagonist injections at a dose of $0.25 \mathrm{mg} /$ day were started either on the sixth day of stimulation or when the leading follicle reached $14 \mathrm{~mm}$. Gonadotropin dosage was adjusted according to ovarian response on day 5. Pelvic ultrasound and endocrine monitoring were performed thereafter. Injections were continued until $\geq 3$ follicles reached $\geq 17 \mathrm{~mm}$ diameter. All participants had high number of follicles measuring $>12 \mathrm{~mm}$ and/or high serum estradiol levels on the day of trigger or had previous history of OHSS. The $\mathrm{GnRH}$ agonist trigger which consisted of SC injection of $1 \mathrm{mg}$ leuprolide acetate (Lucrin, Abbott) was administered. Transvaginal oocyte retrieval was performed 35 - $36 \mathrm{~h}$ after the agonist trigger and 1,500 IU of hCG (Pregnyl, MSD, Turkey) was administered $1 \mathrm{~h}$ after oocyte retrieval. Techniques such as "coasting", cabergoline therapy or cryopreservation of all embryos were used in participants who were identified as being at very high risk of OHSS. Women did not receive 1,500 IU hCG plus luteal phase support who had cryopreservation. Embryo transfer was performed 2 months later according to thaw procedure. Women who underwent fresh embryo transfer received luteal phase support with vaginal micronized progesterone (crinone $90 \mathrm{mg} /$ day; Merck Serono), $50 \mathrm{mg} /$ day IM progesterone (Progestan; Kocak Farma, Turkey) and $6 \mathrm{mg}$ /day oral estradiol hemihydrate tablets (estrofem $2 \mathrm{mg}$ three times daily; Novo-Nordisk, Turkey). A positive serum hCG on day 15 post-oocyte retrieval was defined as positive pregnancy test
Table 1. Patient Characteristics

\begin{tabular}{ll}
\hline Age $(\text { years })^{*}$ & $27.93 \pm 4.36(21-29)$ \\
BMI $\left(\mathrm{kg} / \mathrm{m}^{2}\right)^{*}$ & $24.75 \pm 5.37(19-34)$ \\
Previous cycles** & $1(0-9)$ \\
AFC** & $26(17-34)$ \\
Etiology combined to PCO & \\
$\quad$ Male & $35.9 \%$ \\
$\quad$ Tubal & $4 \%$ \\
\multicolumn{1}{l}{ Endometriosis } & $2 \%$ \\
\hline
\end{tabular}

*Values are mean $\pm S D .{ }^{* *}$ Values are median (minimum - maximum). SD: standard deviation; BMI: body mass index; AFC: antral follicul count.

and evidence of a gestational sac and fetal heart on ultrasound was defined as clinical pregnancy.

The diagnosis of OHSS was based on Golan criteria [13]. Mild OHSS was diagnosed when abdominal distension with or without nausea, vomiting and/or diarrhea was present. Presence of ultrasonographic ascites in addition to the above features revealed moderate OHSS. In severe OHSS, there was clinical evidence of ascites and/or hydrothorax or breathing difficulties with or without hemoconcentration, coagulation abnormalities and diminished renal function.

The two primary outcomes of this study were incidence and severity of OHSS and clinical pregnancy rate.

Data were presented as mean \pm standard deviation (SD) or the median depending on the distribution characteristics. The statistical analysis was carried out using the statistical pack-

Table 2. Ovarian Stimulation Outcomes

\begin{tabular}{|c|c|}
\hline Coasting, n (\%) & $70(79.5 \%)$ \\
\hline Dopamine agonist, $\mathrm{n}(\%)$ & $24(27.2 \%)$ \\
\hline Serum peak estradiol (pg/mL)* & $5,336 \pm 2,341(1,187-19,746)$ \\
\hline Number of follicules $>12 \mathrm{~mm}$ on the day of trigger* & $22 \pm 7(9-51)$ \\
\hline Number of oocytes collected* & $17 \pm 8(0-45)$ \\
\hline Number of metaphase II oocytes* & $11 \pm 5(0-23)$ \\
\hline Number of embryos produced (n)** & $8(1-18)$ \\
\hline Total number of cycles with embryo transfer (ET), n (\%) & $84(95.4 \%)$ \\
\hline Duration of ovarian stimulation (days) ${ }^{* *}$ & $9(6-16)$ \\
\hline Total dose of gonadotropins (IU)* & $1,794 \pm 858(700-4,500)$ \\
\hline Number of embryos transferred (n)** & $1(0-2)$ \\
\hline Number of embryos cryopreserved (n)** & $2(0-10)$ \\
\hline OHSS rate & $12 / 88(13.6 \%)$ \\
\hline Number of cycles with all embryos cryopreserved, n (\%) & $21(23.8 \%)$ \\
\hline Embryo transfer canceled, $\mathrm{n}(\%)$ & $4(4.5 \%)$ \\
\hline Positive pregnancy rate, $\mathrm{n}$ (\% per embryo transfer) & $46 / 84(54.7 \%)$ \\
\hline Clinical pregnancy rate, $\mathrm{n}(\%$ per embryo transfer) & $39 / 84(46.4 \%)$ \\
\hline Miscarriages, $\mathrm{n}(\%$ per cycle with embryo transfer) & $9(10.2 \%)$ \\
\hline
\end{tabular}

*Values are mean \pm SD. ${ }^{* *}$ Values are median (minimum - maximum). SD: standard deviation. 
Table 3. Patient and COS Cycle Characteristics of OHSS Cases

\begin{tabular}{|c|c|c|c|c|c|c|c|c|c|c|}
\hline & Age & BMI & $\begin{array}{l}\text { Gonodotropin } \\
\text { starting } \\
\text { dosage (IU/day) }\end{array}$ & $\begin{array}{l}\text { Total } \\
\text { gonadotropin } \\
\text { dosage }\end{array}$ & Co-treatment & $\begin{array}{l}\text { Number of } \\
\text { follicules } \\
\geq 12 \mathrm{~mm}\end{array}$ & $\begin{array}{l}\text { Peak } \\
\text { estradiol } \\
\text { in } \mathbf{n g} / \mathbf{m L}\end{array}$ & $\begin{array}{l}\text { Number of } \\
\text { oocytes collected } \\
\text { (metaphase 2) }\end{array}$ & $\begin{array}{l}\text { Luteal } \\
\text { hCG } \\
\text { support }\end{array}$ & $\begin{array}{l}\text { Number of } \\
\text { transferred }\end{array}$ \\
\hline Case 1 & 24 & 21 & 125 & 1,075 & Coasting & 15 & 5,000 & $13(11)$ & Yes & 1 \\
\hline Case 2 & 26 & 19 & 150 & 1,237 & Coasting & 21 & 3,817 & $20(11)$ & Yes & 1 \\
\hline Case 3 & 26 & 20 & 200 & 1,800 & Coasting & 18 & 5,894 & $17(12)$ & Yes & 2 \\
\hline Case 4 & 23 & 20 & 225 & 1,950 & Coasting-cabergoline & 30 & 7,012 & $29(18)$ & Yes & 2 \\
\hline Case 6 & 26 & 26 & 150 & 1,125 & Coasting & 20 & 5,090 & $19(10)$ & Yes & 2 \\
\hline Case 7 & 36 & 21 & 125 & 875 & Coasting & 15 & 6,513 & $14(12)$ & Yes & 2 \\
\hline Case 8 & 27 & 23.9 & 125 & 725 & Coasting & 29 & 5,985 & $27(18)$ & No & Freeze \\
\hline Case 9 & 31 & 31 & 150 & 2,160 & Coasting-cabergoline & 35 & 8,644 & $8(3)$ & No & Freeze \\
\hline Case 10 & 26 & 23 & 125 & 1,250 & Coasting & 42 & 5,570 & $24(21)$ & Yes & Freeze \\
\hline
\end{tabular}

BMI: body mass index; hCG: human chorionic gonadotropin.

age for social sciences (SPSS). Significance was defined as P $<0.05$. For comparison of the continuous variables, the Student's $t$-test was performed where the parametric non-continuous variables were compared using Mann-Whitney U test.

\section{Results}

A total of 88 cycles were undertaken in the study. Sixty-five women received the GnRH agonist trigger plus 1,500 IU hCG protocol. Twenty-three women received GnRH agonist trigger without hCG. All participants either had PCOS which was diagnosed according to the Rotterdam criteria [14] or isolated PCO morphology [15]. Forty-seven (53.4\%) patients had their first IVF cycle. The baseline patient characteristics are shown in Table 1. Sixty-three (71.5\%) women underwent fresh embryo transfer. Fresh embryo transfer was canceled in $21(23.8 \%)$ women (two due to endometrial polyp, eight due to premature rise in progesterone levels, and 11 due to signs of OHSS) and embryo transfer was canceled for four (4.5\%) women. Positive pregnancy test rate was $54.7 \%$ and clinical pregnancy rate was $46.4 \%$. Ovarian stimulation characteristics and outcomes are presented in Table 2. A total of 12 (13.6\%) patients developed OHSS. Eleven of these developed OHSS despite co-treatment with either coasting and/or cabergoline. Eight of these women had fresh embryo transfer and the other four had all their embryos cryopreserved. Of four patients whose embryos were cryopreserved, embryo transfer in one patient was canceled after administration of 1,500 IU hCG because of early signs of OHSS. The other three patients developed OHSS despite 1,500 IU hCG was not administered. Patient and COS cycle characteristics of OHSS cases were summarized in Table 3. Clinical characteristics of OHSS cases are presented in Table 4. We compared female age, BMI, numbers of follicles $>12$ $\mathrm{mm}$, on the day of trigger and peak serum E2 levels between the 12 women who had OHSS and the 76 women who did not develop the syndrome. The number of follicles measuring $>12$ $\mathrm{mm}$ on the day of trigger was significantly different between groups (Table 5).

\section{Discussion}

Development of OHSS has been an unsolved problem since the introduction of ovarian stimulation for assisted reproduction. Several methods and markers have been defined to identify the high-risk patients and different preventive strategies have been developed. Previous studies besides normo-responder patients even in high-risk patients on $\mathrm{GnRH}$ agonist triggering plus 1,500 IU hCG supported the view that complete prevention of OHSS with good or even nearly excellent pregnancy rates was possible $[16,17]$. However, their view seems to be not shared by Seyhan and colleagues [12].

Studies on GnRH agonist trigger and luteal support with a bolus of 1,500 IU hCG have gained interest in patients at high risk for OHSS. Engmann et al [4] in their prospective randomized study have shown that the use of a GnRH agonist to trigger oocyte maturation after co-treatment with GnRH antagonist combined with adequate luteal phase and early pregnancy estradiol and progesterone supplementation in patients with PCOS or PCO morphology is effective in reducing the risk of OHSS without adversely affecting implantation rates.

GnRH agonist trigger followed by a modified luteal phase support with one bolus of 1,500 IU hCG was developed by Humaidan et al [16].

This approach was tested in a group of normo-responder subjects in previous studies conducted by also Humaidan et al $[16,18,19]$.

Two previous case series with small cohort of patients reported complete prevention of early OHSS when the GnRH agonist trigger plus $1,500 \mathrm{IU}$ hCG support protocol was employed in patients with high risk of OHSS $[10,11]$. Recently, 
Table 4. Clinical Characteristics of OHSS Cases

\begin{tabular}{lllllll}
\hline & OHSS & OHSS type & WBC $(\mu \mathrm{L})$ & Hct $(\%)$ & Paracentesis (amount drained) & Pregnancy \\
\hline Case 1 & Late & Severe & 19,400 & 0.46 & Once $(1 \mathrm{~L})$ & Yes \\
Case 2 & Early & Severe & 20,100 & 0.43 & Once $(1.4 \mathrm{~L})$ & No \\
Case 3 & Early & Severe & 24,000 & 0.43 & Once $(1.8 \mathrm{~L})$ & No \\
Case 4 & Early & Severe & 20,800 & 0.41 & Once $(1 \mathrm{~L})$ & Yes \\
Case 5 & Late & Severe & 28,000 & 0.49 & Twice $(2.9 \mathrm{~L})$ & Yes \\
Case 6 & Early & Severe & 19,400 & 0.43 & Once $(1.5 \mathrm{~L})$ & Yes \\
Case 7 & Early & Severe & 20,100 & 0.50 & Once $(1.8 \mathrm{~L})$ & No \\
Case 8 & Early & Severe & 24,000 & 0.50 & Three times $(3 \mathrm{~L})$ & Embryos freezed \\
Case 9 & Early & Severe & 20,800 & 0.45 & Once $(0.5 \mathrm{~L})$ & Embryos freezed \\
Case 10 & Early & Moderate & 21,000 & 0.42 & Once $(0.5 \mathrm{~L})$ & Embryos freezed \\
Case 11 & Early & Moderate & 21,900 & 0.36 & Twice $(1 \mathrm{~L})$ & Embryos freezed \\
Case 12 & Early & Severe & 31,700 & 0.51 & Three times $(5.5 \mathrm{~L})$ & Yes \\
\hline
\end{tabular}

OHSS: ovarian hyperstimulation syndrome; WBC: white blood cell; Hct: hematocrit.

Iliodromiti et al [17] in their three-center retrospective analysis including 275 patients at high risk of developing OHSS showed high clinical pregnancy rates and very low OHSS rates who received a GnRH agonist trigger followed by a bolus of 1,500 IU hCG $1 \mathrm{~h}$ after oocyte retrieval. But discordance between baseline characteristics and ovarian response of the study population was claimed. The study population seemed to belong to a moderate responder group rather than a highrisk group. The severe OHSS rate was reported as $0.72 \%$ and the discordance between baseline characteristics and ovarian response of the study population gives rise to questions about the study population and/or medication administered [20, 21].

However, the last words seems not to have been said on the issue in high-risk patients because there is a challenging study which did not claim a low incidence of OHSS with this modified protocol [12], even the study has been criticized by authors experienced on the issue.

The current retrospective study was performed in patients with PCOS or PCO morphology. Patients received GnRH agonist trigger plus 1,500 IU hCG in GnRH antagonist cycles. Our pregnancy rates were comparable with previous studies, but severe OHSS incidence was higher. A total of $12(13.6 \%)$ women developed OHSS, and $10(11.3 \%)$ of these women had severe OHSS (two late and eight early OHSS). Eight of them had fresh embryo transfer and the other four had all their embryos cryopreserved. Eleven women developed OHSS despite co-treatment with either coasting and/or cabergoline.

A previous retrospective study by Seyhan et al [12] reported $26 \%$ of severe OHSS in high-risk patients, following GnRH agonist trigger and modified luteal phase support with one bolus of 1,500 IU hCG. Their series included 23 patients and 1,500 IU hCG was administered $1 \mathrm{~h}$ before oocyte retrieval; this may be a reason for high incidence of OHSS. There is also discrepancy between the follicular count and the actual number of oocytes they collected in their series. Especially the numbers of follicles $\geq 12 \mathrm{~mm}$ were 11 and 15 in OHSS cases 1 and 2, while the numbers of metaphase 2 oocytes collected in the same patients were 41 and 32. Explaining this discrepancy by collecting mature oocytes from follicles $<10 \mathrm{~mm}$ seems unreasonable. Seyhan et al [12] also suggested upper cut-off value of $\geq 18$ follicles measuring 10 - $14 \mathrm{~mm}$ on the day of agonist trigger as risk factor for OHSS.

In the present study, we found significantly higher number of follicles $>12 \mathrm{~mm}$ in patients who developed OHSS. The mean number of follicles measuring $>12 \mathrm{~mm}$ was 27.5 in OHSS group, while it was 21.6 in non-OHSS group. Hu-

Table 5. Comparison of Women Who Did and Did Not Develop OHSS

\begin{tabular}{llll}
\hline & OHSS group & Non-OHSS group & P-value \\
\hline Age in years & $27.5 \pm 3.7$ & $27.9 \pm 4.4$ & 0.48 \\
BMI $\left(\mathrm{kg} / \mathrm{m}^{2}\right)$ & $23.5 \pm 4.41$ & $24.9 \pm 2.4$ & 0.36 \\
Number of follicles $>$ 12 mm on the day of trigger & $27.5 \pm 11.1$ & $21.6 \pm 6.4$ & 0.005 \\
Coasting $(\%)$ & $10 / 12(83.3)$ & $58 / 76(76.3)$ & 0.90 \\
Cabergoline (\%) & $3 / 12(25)$ & $21 / 76(27.6)$ & 0.60 \\
Total FSH dosage & $1,492 \pm 525$ & $1,846 \pm 890$ & 0.18 \\
Peak estradiol in $\mathrm{pg} / \mathrm{mL}$ & $5,598 \pm 1,506$ & $5,295 \pm 2,450$ & 0.27 \\
\hline
\end{tabular}

OHSS: ovarian hyperstimulation syndrome; BMI: body mass index. 
maidan et al recommended "freeze all" policy in patients with $>25$ follicles $\geq 11 \mathrm{~mm}$, to avoid any risk of OHSS development [22]. In our study, three patients developed OHSS despite luteal support with 1,500 IU hCG was canceled. Number of follicles measuring $\geq 12 \mathrm{~mm}$ in these patients was 28 , 35 and 51. Freeze-all policy did not attenuate OHSS in these three patients. Two of them developed early severe and one of them developed moderate early OHSS. In the light of previous publications suggesting freezing all embryos would prevent OHSS development; we were surprised to see our first patient who developed OHSS after having all her embryos frozen. She referred to our center with severe abdominal distention and dyspnea 4 days after oocyte retrieval. Saline infusion was administered, abdominal drainage was performed and dopamine agonist (cabergoline, $0.5 \mathrm{mg} /$ day) treatment was started. After 4 days of hospitalization, her condition improved and was discharged from hospital. This experience showed that high-risk patients should be informed about the signs of OHSS even after freezing all embryos. Other two cases who also had freezing all embryos and did not have any hCG exposure referred to our center within 2 days after oocyte retrieval and were managed successfully [23]. Recently, Fatemi et al [24] reported two cases of severe OHSS after GnRH agonist trigger in a GnRH antagonist protocol without the administration of any hCG for luteal phase support. The underlying mechanism of development of OHSS in the absence of hCG is obscure.

Eighty-eight PCO patients with high risk from a single center make our study one of the studies involving maximum number of patients of its kind. However, there are some limitations of our study. The retrospective design of the current study design was a limitation. However, in previous prospective studies [22] patients with relatively low OHSS risk $(>12$ $\mathrm{mm}, 15-25$ follicles at the day of hCG) compared with the current study were divided into two groups GnRH agonist vs. hCG trigger. But the participants in the current study included patients with high risk of OHSS and PCO morphology (25 and over $>12 \mathrm{~mm}$ follicle) and due to the possibility to face lifethreatening OHSS cases, we found it not very convenient as ethical to do the same in a controlled and prospective study.

A patient with an estradiol level of $1,187 \mathrm{pg} / \mathrm{mL}$ was enrolled in the study. Inclusion criteria of the current study were 14 and more follicles $>12 \mathrm{~mm}$ in diameter or high estradiol levels or history of previous OHSS. The patient had previously undergone severe OHSS and despite the evacuation of pregnancy, she was hospitalized for 4 months. In the current study for the patients with high risk for OHSS, methods such as cabergoline and/or coasting were used necessarily. In other studies, these methods were also used. Although this method was used OHSS frequency was $13.6 \%$ in the current study. We showed in the study that we did not observe the low OHSS rates with $\mathrm{GnRH}$ agonist trigger as claimed in previous studies $[17,22]$. Although we used coasting and cabergoline in addition, this is the crucial point of our study we want to draw attention to.

In conclusion, previous reports suggested that GNRH agonist triggering plus low-dose hCG supplementation protocol is effective in reducing the OHSS incidence but we support the findings of Seyhan et al [12], who reported that this modified protocol does not eliminate the OHSS risk. We also conclude that "freeze all" policy and not administering hCG does not always eliminate OHSS development. We recommend that high-risk patients should be informed about the signs of OHSS even after employing "freeze-all" policy. Identifying high-risk women for OHSS at the patient's first visit and developing appropriate stimulation protocol for complete elimination of OHSS are important.

\section{Acknowledgement}

We thank all patients and staff of the study center (Fuat Ali, Ahmet Salvarci, Sukru Uzman, Melek Kaya, and Betul Ince).

\section{Funding}

No funding was saught for this report.

\section{Conflict of Interest}

None.

\section{References}

1. Delvigne A, Rozenberg S. Epidemiology and prevention of ovarian hyperstimulation syndrome (OHSS): a review. Hum Reprod Update. 2002;8(6):559-577.

2. Lee TH, Liu CH, Huang CC, Wu YL, Shih YT, Ho HN, Yang YS, et al. Serum anti-Mullerian hormone and estradiol levels as predictors of ovarian hyperstimulation syndrome in assisted reproduction technology cycles. Hum Reprod. 2008;23(1):160-167.

3. Esinler I, Bayar U, Bozdag G, Yarali H. Outcome of intracytoplasmic sperm injection in patients with polycystic ovary syndrome or isolated polycystic ovaries. Fertil Steril. 2005;84(4):932-937.

4. Engmann L, DiLuigi A, Schmidt D, Nulsen J, Maier $\mathrm{D}$, Benadiva C. The use of gonadotropin-releasing hormone $(\mathrm{GnRH})$ agonist to induce oocyte maturation after cotreatment with $\mathrm{GnRH}$ antagonist in high-risk patients undergoing in vitro fertilization prevents the risk of ovarian hyperstimulation syndrome: a prospective randomized controlled study. Fertil Steril. 2008;89(1):84-91.

5. Griesinger G, von Otte S, Schroer A, Ludwig AK, Diedrich K, Al-Hasani S, Schultze-Mosgau A. Elective cryopreservation of all pronuclear oocytes after GnRH agonist triggering of final oocyte maturation in patients at risk of developing OHSS: a prospective, observational proof-ofconcept study. Hum Reprod. 2007;22(5):1348-1352.

6. Babayof R, Margalioth EJ, Huleihel M, Amash A, ZylberHaran E, Gal M, Brooks B, et al. Serum inhibin A, VEGF and TNFalpha levels after triggering oocyte maturation with GnRH agonist compared with HCG in women with polycystic ovaries undergoing IVF treatment: a prospective randomized trial. Hum Reprod. 2006;21(5):1260- 
1265 .

7. Orvieto R. Can we eliminate severe ovarian hyperstimulation syndrome? Hum Reprod. 2005;20(2):320-322.

8. Kol S. Prediction of ovarian hyperstimulation syndrome: why predict if we can prevent! Hum Reprod. 2003;18(7):1557-1558.

9. Kol S, Itskovitz-Eldor J. Severe OHSS: yes, there is a strategy to prevent it! Hum Reprod. 2000;15(11):22662267.

10. Humaidan P. Luteal phase rescue in high-risk OHSS patients by GnRHa triggering in combination with low-dose HCG: a pilot study. Reprod Biomed Online. 2009;18(5):630-634.

11. Radesic B, Tremellen K. Oocyte maturation employing a GnRH agonist in combination with low-dose hCG luteal rescue minimizes the severity of ovarian hyperstimulation syndrome while maintaining excellent pregnancy rates. Hum Reprod. 2011;26(12):3437-3442.

12. Seyhan A, Ata B, Polat M, Son WY, Yarali H, Dahan MH. Severe early ovarian hyperstimulation syndrome following GnRH agonist trigger with the addition of $1500 \mathrm{IU}$ hCG. Hum Reprod. 2013;28(9):2522-2528.

13. Golan A, Ron-el R, Herman A, Soffer Y, Weinraub Z, Caspi E. Ovarian hyperstimulation syndrome: an update review. Obstet Gynecol Surv. 1989;44(6):430-440.

14. Revised 2003 consensus on diagnostic criteria and longterm health risks related to polycystic ovary syndrome. Fertil Steril. 2004;81(1):19-25.

15. Balen AH, Laven JS, Tan SL, Dewailly D. Ultrasound assessment of the polycystic ovary: international consensus definitions. Hum Reprod Update. 2003;9(6):505-514.

16. Humaidan $\mathrm{P}$, Bungum L, Bungum M, Yding Andersen C. Rescue of corpus luteum function with peri-ovulatory HCG supplementation in IVF/ICSI GnRH antagonist cycles in which ovulation was triggered with a GnRH agonist: a pilot study. Reprod Biomed Online.
2006;13(2):173-178.

17. Iliodromiti S, Blockeel C, Tremellen KP, Fleming R, Tournaye H, Humaidan P, Nelson SM. Consistent high clinical pregnancy rates and low ovarian hyperstimulation syndrome rates in high-risk patients after $\mathrm{GnRH}$ agonist triggering and modified luteal support: a retrospective multicentre study. Hum Reprod. 2013;28(9):2529-2536.

18. Humaidan P, Ejdrup Bredkjaer $\mathrm{H}$, Westergaard LG, Yding Andersen C. 1,500 IU human chorionic gonadotropin administered at oocyte retrieval rescues the luteal phase when gonadotropin-releasing hormone agonist is used for ovulation induction: a prospective, randomized, controlled study. Fertil Steril. 2010;93(3):847-854.

19. Humaidan P, Quartarolo J, Papanikolaou EG. Preventing ovarian hyperstimulation syndrome: guidance for the clinician. Fertil Steril. 2010;94(2):389-400.

20. Gurbuz AS, Deveer R, Kucuk M. GnRH agonist triggering in high-risk patient: Hum Reprod. 2014;29(7):1598.

21. Ilidromiti S, Nelson S M, et al. Reply: GnRH agonist triggering in high-risk patient. Hum Reprod. 2014;29(7):1598-1599.

22. Humaidan P, Thomsen LH, Alsbjerg B. GnRHa trigger and modified luteal support with one bolus of hCG should be used with caution in extreme responder patients. Hum Reprod. 2013;28(9):2593-2594.

23. Gurbuz AS, Gode F, Ozcimen N, Isik AZ. Gonadotrophin-releasing hormone agonist trigger and freeze-all strategy does not prevent severe ovarian hyperstimulation syndrome: a report of three cases. Reprod Biomed Online. 2014;29(5):541-544.

24. Fatemi HM, Popovic-Todorovic B, Humaidan P, Kol S, Banker M, Devroey P, Garcia-Velasco JA. Severe ovarian hyperstimulation syndrome after gonadotropinreleasing hormone $(\mathrm{GnRH})$ agonist trigger and "freezeall" approach in GnRH antagonist protocol. Fertil Steril. 2014;101(4):1008-1011. 